\title{
简便合成新型四桥联双杯[4]管道和 1,2-3,4-双桥联杯[4]芳烃
}

\author{
张瑛美 ${ }^{a}$ 杨发福*,a,b 白小艳 ${ }^{a}$ 刘志强 郭红玉 ${ }^{a}$ \\ ( ${ }^{a}$ 福建师范大学化学与材料学院 福州 350007) \\ ${ }^{b}$ 福建省高分子材料重点实验室 福州 350007)
}

\begin{abstract}
摘要 报道了一种间接缩合法高产率简便合成新型四桥联双杯[4]管道和 1,2-3,4-双桥联杯[4]芳烃. 杯[4]二酰肼衍生物 2 与丁二酸酩发生开环反应，以 $95 \%$ 的产率得到杯 [4]羧酸衍生物 4 . 化合物 4 在 $\mathrm{DCC} / \mathrm{DMAP} / \mathrm{CH}_{2} \mathrm{Cl}_{2}$ 体系中发生分子间 自缩合反应，以 $90 \%$ 产率得到新型四桥联双杯 [4]管道 6. 改用高度稀释的条件则以 $26 \%$ 的产率得到新型 1,2-3,4-双桥联 杯 [4]芳烃 5. 新化合物的结构与构象经元素分析、红外、质谱、核磁共振谱等表征证实.
\end{abstract}

关键词 双杯[4]管道; 双桥联杯 [4]芳烃; 合成

\section{Convenient Synthesis of Novel Tetra-bridged Biscalix[4]tube and 1,2-3,4-Bis-bridged Calix[4]arene}

\author{
Zhang, Yingmei $^{a} \quad$ Yang, Fafu $^{*, a, b} \quad$ Bai, Xiaoyan $^{a} \quad$ Liu, Zhiqiang ${ }^{a} \quad$ Guo, Hongyu ${ }^{a}$ \\ ( ${ }^{a}$ College of Chemistry and Materials, Fujian Normal University, Fuzhou 350007) \\ ( ${ }^{b}$ Fujian Key Laboratory of Polymer Materials, Fuzhou 350007)
}

\begin{abstract}
This paper described the indirect and convenient syntheses of novel tetra-bridged calix[4]tube and 1,2-3,4-bis-bridged calix[4]arene in high yields. By the ring-opening reaction of calix[4]-bis-hydrazide derivative 2 with succinic anhydride, calix[4]arene carboxyl derivative 4 was obtained in $95 \%$ yield. Under the DCC/DMAP/ $\mathrm{CH}_{2} \mathrm{Cl}_{2}$ system, novel tetra-bridged calix[4]tube $\mathbf{6}$ was prepared by the intermolecular self-condensation of compound $\mathbf{4}$ in $90 \%$ yield. Under the same system in high diluted condition, novel 1,2-3,4-bis-bridged calix[4]arene 5 was synthesized in $26 \%$ yield as by-product. The structures and conformations of new compounds were characterized by elemental analyses, IR, ESI-MS, ${ }^{1} \mathrm{H}$ NMR, etc.

Keywords biscalix[4]tube; bis-bridged calix[4]arene; synthesis
\end{abstract}

杯芳烃是有机超分子领域广受瞩目的人工合成主 体分子平台, 通过适当的化学修饰可以构筑对特定客体 具有高识别能力的新型主体分子. 双杯芳烃是其衍生物 中重要的一类, 通过两个杯芳烃单元的协同作用可产生 单个杯芳烃无法具备的特殊客体识别能力 ${ }^{[1 \sim 3]}$. 一般地, 通过双官能团试剂与杯芳烃直接缩合, 可得到结构对称 的双杯芳烃, 但由于多羟基的桥联方式多样, 产率一般 不高, 分离较复杂 ${ }^{[4 \sim 6]}$. 另一方面, 通过先于杯芳烃单 元引入活性官能团, 再进一步与另一杯芳烃单元反应可 得到含两种不同结构单元的双杯芳烃, 由于步骤较为复 杂, 这种间接合成法报道较少 ${ }^{[7-10]}$. 如果用四条桥联链
将两个杯 [4]芳烃单元相连，可得到双杯 [4]管道 (calix[4]tube)，这种管状空腔可望在复杂客体配合和客 体通道模拟方面具有优势. 但由于杯[4]芳烃单元多羟 基桥联的复杂性，这类分子报道较少，一般产率很 低 ${ }^{[1 \sim 13]}$. 本文中, 我们设计并合成了一种新型氮杂四桥 联双杯[4]管道 5, 几乎没有副产物, 分离简便, 产率很 高. 通过改变反应条件，还得到了一种新型结构的 1,2-3,4-双桥联杯[4]芳烃 6, 产率中等, 为合成这类结构 复杂的主体分子提供了新的思路. 合成路线如 Scheme 1 所示.

\footnotetext{
*E-mail: yangfafu@fjnu.edu.cn

Received July 8, 2012; revised August 25, 2012; published online August 30, 2012.

Project supported by the National Natural Science Foundation of China (No. 20402002), the Natural Science Foundation of Fujian Province (No. 2011J01031), the Project of Fujian Provincial Department of Education (No. JA11044) and the Program for Excellent Young Researchers in University of Fujian Province (No. JA10056).

国家自然科学基金(No. 20402002)、福建省自然科学基金(No. 2011J01031)、福建省高校杰出青年科研人才培育计划(No. JA10056)和福建省教育厅重 点(No. JA11044)资助项目.
} 

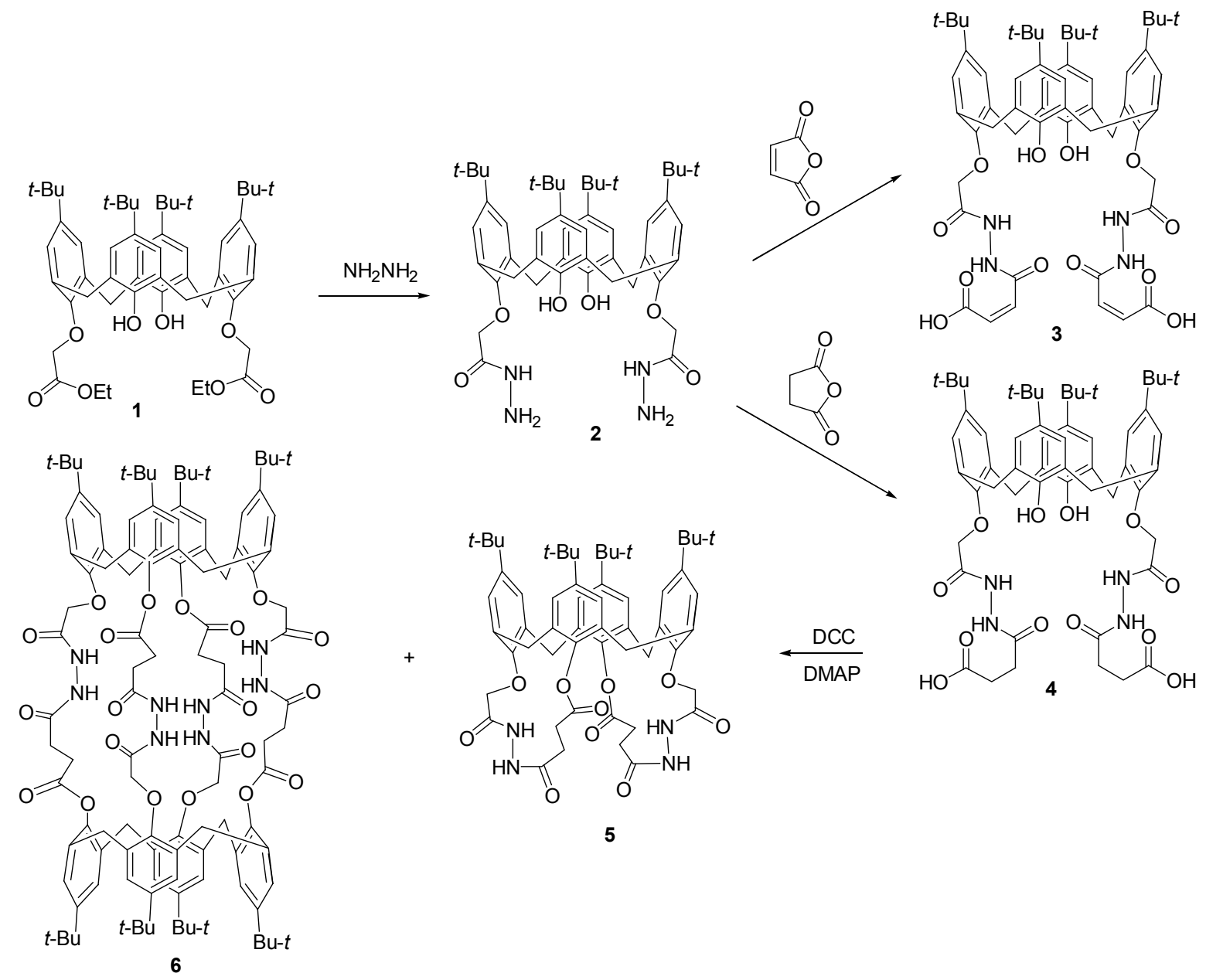

\section{Scheme 1}

\section{1 结果与讨论}

文献中，四桥联双杯 [4]管道一般通过双官能团试 剂与两个杯 [4]芳烃单元直接缩合得到, 由于桥联选择 性较低, 产率一般较低, 分离较为困难 ${ }^{[11,12]}$. 我们曾通 过制备末端含氯甲基的四取代杯 [4]芳烃衍生物与杯 [4] 芳烃反应得到双杯 [4]管道，这种间接合成方法虽步骤 较多, 但副产物少, 产率较高, 分离难度不大 ${ }^{[13]}$. 本文 中, 我们借鉴间接法的思路, 先高产率合成杯 [4]二酰肼 2, 然后分别与顺丁烯二酸酐和丁二酸酐发生开环反应, 得到末端含羒基的杯 [4]衍生物 3 和 $\mathbf{4}$. 在 DCC/DMAP/ $\mathrm{CH}_{2} \mathrm{Cl}_{2}$ 体系中，化合物 4 直接发生分子间自身缩合得到 新型四桥联双杯 [4]管道 6, 反应过程中 TLC 检测几乎没 有发现其它副产物, 产率高达 $90 \%$. 如果该反应在高度 稀释条件下进行, 则出现分子内缩合副产物, 为 1,2-3,4双桥联杯 [4]芳烃 5, 产率 26\%. 有趣的是, 杯 [4]丁二酸 衍生物 4 可以很好地进行上述反应，但杯[4]顺丁烯二酸 衍生物 3 却在同样条件下不发生类似反应, 改变反应条 件, 也没有分离出结构确定的产物, 这可能是顺丁烯酸
的顺式刚性结构的影响，具体原因有待深入探究. 我们 曾尝试通过化合物 4 的羧基酰氯化再进行缩合反应，发 现产物复杂得多. 我们也曾试图在 DCC/DMAP/ $/ \mathrm{CH}_{2} \mathrm{Cl}_{2}$ 的反应条件下用化合物 $\mathbf{4}$ 与其它醇类如乙醇、丙醇等发 生类似酯化缩合反应，却发现得到的产物依然是四桥联 双杯 [4]管道 6 为主, 没有分离得到其它缩合产物. 我们 推测这可能是由于化合物 $\mathbf{4}$ 中柔性链含的氨基、羧基和 酚差基等易于互相形成氢键，在缩合前就易以 “ $1+1$ ” 的方式通过氢键形成稳定的超分子结构, 并最终高产率 得到分子间自缩合产物 6. 这些结果说明桥联链的结构 对缩合反应具有重要的影响. 值得指出的是, 本文得到 的四桥联双杯 [4]管道 $\mathbf{6}$ 是文献报道中产率最高的. 得到 的具有不对称链的新型 1,2-3,4-双桥联杯 [4]芳烃 5 也是 文献少见报道的类型 ${ }^{[14 \sim 16]}$.

所有新化合物均经元素分析、红外、质谱、核磁共 振谱图等表征证明为 Scheme 1 所示的结构与构象. ESI-MS 谱图中出现相应的分子离子基峰, 化合物 6 为 $1912.1\left(\mathrm{M}^{+}\right)$, 化合物 5 为 $956.7\left(\mathrm{M}^{+}\right)$, 说明它们分别是 分子间和分子内的缩合产物. 核磁氢谱中，化合物 6 的 
$\mathrm{ArCH}_{2} \mathrm{Ar}$ 为 $1: 1$ 的一对双峰, 说明其采取 1,3-桥联模式 且为杯式构象, 与文献的报道也是一致的. 化合物 5 的 $\mathrm{ArCH}_{2} \mathrm{Ar}$ 也为 $1: 1$ 的一对双峰, 没有细分为 $\mathrm{ABAB}$ 模 式, 这可能是桥联链上官能团较为相似的结果. 这两种 新型双杯 [4]管道和分子内双桥联杯[4]芳烃的性能正在 研究当中.

\section{2 结论}

本文通过间接缩合法高产率简便合成了新型四桥 联双杯 [4]管道和 1,2-3,4-双桥联杯 [4]芳烃. 杯[4]二酰肼 衍生物 2 与丁二酸酕或顺丁烯二酸䣶发生开环反应，以 95\%的产率得到杯 [4]羧酸衍生物 $\mathbf{3}$ 和 $\mathbf{4}$. 化合物 $\mathbf{4}$ 在 $\mathrm{DCC} / \mathrm{DMAP} / \mathrm{CH}_{2} \mathrm{Cl}_{2}$ 体系中发生分子间自缩合反应，以 $90 \%$ 产率得到新型四桥联双杯 [4]管道 6. 改用高度稀释 的条件则以 $26 \%$ 的产率得到新型 1,2-3,4-双桥联杯[4]芳 烃 5. 化合物 3 不发生类似反应, 说明桥联链的结构对 缩合反应有重要的影响作用.

\section{3 实验部分}

\section{1 仪器与试剂}

Varian Unity 500 (500 MHz)核磁共振仪; DECAX-30000 LCQ Deca XP ESI-MS 质谱仪(甲醇作溶剂); AVATAR360 型红外光谱仪; Reichert 7905 熔点仪(温度 未校正); Vario EL III 自动元素分析仪. 二氯甲烷经水洗 后氯化钙干燥使用, 水合肼重蒸后使用, 其它试剂为分 析纯直接使用. 对叔丁基杯 [4]芳烃二酯衍生物 1 及其肼 解衍生物 2 按文献方法合成 ${ }^{[17,18]}$.

\section{2 合成}

\subsection{1 杯[4]顺丁烯二酸酐开环衍生物 3 的合成}

冰浴 $\left(-5 \sim 0{ }^{\circ} \mathrm{C}\right)$ 下, 在 $50 \mathrm{~mL}$ 烧瓶中加入杯 $[4]$ 二酯 二酰胇衍生物 $2(0.60 \mathrm{~g}, 0.76 \mathrm{mmol})$, 顺丁烯二酸䣶 $(0.20$ $\mathrm{g}, 2 \mathrm{mmol}$ )和 $25 \mathrm{~mL}$ 氯仿. 搅拌反应 $25 \mathrm{~h}$, 用 TLC 跟踪 检测至原料消失, 常温下再摚拌 $0.5 \mathrm{~h}$, 加入 $15 \mathrm{~mL}$ 温水 摚拌, 趁热分液, 有机层室温减压蒸去大部分溶剂, 残 余物浓缩至 $2 \sim 3 \mathrm{~mL}$, 再加入适量甲醇析出白色沉淀. 沉淀物用 $10 \mathrm{~mL}$ 甲醇-水 $(V: V=1: 1)$ 重结晶, 干燥后 得到白色粉末固体 $3,0.71 \mathrm{~g}$, TLC 检测为一个点, 产率 95\%. m.p. $226 \sim 229{ }^{\circ} \mathrm{C} ;{ }^{1} \mathrm{H}$ NMR $\left(500 \mathrm{MHz}, \mathrm{CDCl}_{3}\right) \delta$ : $0.96\left(\mathrm{~s}, 18 \mathrm{H}, \mathrm{C}\left(\mathrm{CH}_{3}\right)_{3}\right), 1.25$ (s, $\left.18 \mathrm{H}, \mathrm{C}\left(\mathrm{CH}_{3}\right)_{3}\right), 3.43$ (d, $\left.J=15.0 \mathrm{~Hz}, 4 \mathrm{H}, \mathrm{ArCH}_{2} \mathrm{Ar}\right), 4.19(\mathrm{~d}, J=15.0 \mathrm{~Hz}, 4 \mathrm{H}$, $\left.\mathrm{ArCH}_{2} \mathrm{Ar}\right), 4.78$ (s, $\left.4 \mathrm{H}, \mathrm{OCH}_{2} \mathrm{CO}\right), 6.33$ (s, $\left.2 \mathrm{H}, \mathrm{COCH}\right)$, $6.44(\mathrm{~s}, 2 \mathrm{H}, \mathrm{COCH}), 6.70(\mathrm{~s}, 2 \mathrm{H}, \mathrm{OH}), 6.86(\mathrm{~s}, 4 \mathrm{H}, \mathrm{ArH})$, $6.93(\mathrm{~s}, 4 \mathrm{H}, \operatorname{ArH}), 7.85(\mathrm{~s}, 4 \mathrm{H}, \mathrm{NH}), 12.12(\mathrm{bs}, 2 \mathrm{H}$, $\mathrm{COOH})$; IR $(\mathrm{KBr}) v$ : $3417(\mathrm{OH}$ and $\mathrm{NH}), 1713(\mathrm{C}=\mathrm{O})$, $1631(\mathrm{C}=\mathrm{O}) \mathrm{cm}^{-1}$; MS m/z (\%): $988.6\left(\mathrm{M}^{+}, 100\right)$. Anal. calcd for $\mathrm{C}_{56} \mathrm{H}_{68} \mathrm{~N}_{4} \mathrm{O}_{12}$ : C 67.97, H 6.88, N 5.67; found C 67.92, H 6.85, N 5.61.

\subsection{2 杯[4]丁二酸酐开环衍生物 4 的合成}

冰浴 $\left(-5 \sim 0{ }^{\circ} \mathrm{C}\right)$ 下, 在 $50 \mathrm{~mL}$ 烧瓶中加入杯 $[4]$ 二酯 二酰肼衍生物 $2(0.60 \mathrm{~g}, 0.76 \mathrm{mmol})$, 顺丁烯二酸䣶 $(0.20$ $\mathrm{g}, 2 \mathrm{mmol}$ )和 $25 \mathrm{~mL}$ 氯仿. 搅拌反应 $20 \mathrm{~h}$, 用 TLC 跟踪 检测至原料消失, 常温下再搅拌 $0.5 \mathrm{~h}$, 加入 $15 \mathrm{~mL}$ 温水 摚拌, 趁热分液, 有机层室温减压蒸去大部分溶剂, 残 余物浓缩至 $2 \sim 3 \mathrm{~mL}$, 再加入适量甲醇析出白色沉淀. 沉淀物用 $10 \mathrm{~mL}$ 甲醇-水 $(V: V=1: 1)$ 重结晶, 干燥后 得到白色粉末固体 4, $0.71 \mathrm{~g}$, TLC 检测为一个点, 产率 95\%. m.p. $215 \sim 217{ }^{\circ} \mathrm{C} ;{ }^{1} \mathrm{H}$ NMR $\left(500 \mathrm{MHz}, \mathrm{CDCl}_{3}\right) \delta$ : $1.07\left(\mathrm{~s}, 18 \mathrm{H}, \mathrm{C}\left(\mathrm{CH}_{3}\right)_{3}\right), 1.24\left(\mathrm{~s}, 18 \mathrm{H}, \mathrm{C}\left(\mathrm{CH}_{3}\right)_{3}\right), 2.61(\mathrm{~s}$, $\left.4 \mathrm{H}, \mathrm{CH}_{2} \mathrm{CO}\right), 2.72\left(\mathrm{~s}, 4 \mathrm{H}, \mathrm{CH}_{2} \mathrm{CO}\right), 3.42(\mathrm{~d}, J=15.0 \mathrm{~Hz}$, $\left.4 \mathrm{H}, \mathrm{ArCH}_{2} \mathrm{Ar}\right), 4.19$ (d, J=15.0 Hz, 4H, $\left.\mathrm{ArCH}_{2} \mathrm{Ar}\right), 4.71$ (s, 4H, $\mathrm{OCH}_{2} \mathrm{CO}$ ), 6.95 (s, 4H, ArH), 7.05 (s, 4H, ArH), 8.05 (s, 2H, OH), 9.65 (bs, 4H, NH), 11.4 (s, 2H, $\mathrm{COOH})$; IR (KBr) v: $3423(\mathrm{OH}, \mathrm{NH}), 1714(\mathrm{C}=\mathrm{O}), 1638(\mathrm{C}=\mathrm{O})$ $\mathrm{cm}^{-1}$; MS m/z (\%): $992.1\left(\mathrm{M}^{+}, 100\right)$. Anal. calcd for $\mathrm{C}_{56} \mathrm{H}_{72} \mathrm{~N}_{4} \mathrm{O}_{12}$ : C 67.72, H 7.31, N 5.64; found C 67.65, H $7.38, \mathrm{~N} 5.51$.

\subsection{3四桥联双杯 [4]管道 6 的合成}

氮气保护下，向干燥的 $50 \mathrm{~mL}$ 三口瓶中依次加入 10 $\mathrm{mL}$ 干燥二氯甲烷，杯[4]芳烃衍生物 $4(0.25 \mathrm{~g}, 0.25$ $\mathrm{mmol}), N, N^{\prime}$-二环已基碳二亚胺(DCC) $(0.11 \mathrm{~g}, 0.53$ $\mathrm{mmol}$ )和 4-二甲氨基吡啶(DMAP) $(0.005 \mathrm{~g}, 0.04 \mathrm{mmol}$ ), 室温下反应 $10 \mathrm{~h}$, 用 TLC 检测跟踪至原料消失. 加入 10 $\mathrm{mL}$ 蒸馏水搅拌, 静置分液, 有机层干燥, 减压浓缩至 $2 \sim 3 \mathrm{~mL}$, 加入 $10 \mathrm{~mL}$ 甲醇得到白色沉淀. 沉淀用 $15 \mathrm{~mL}$ 二氯甲烷一甲醇 $(V: V=1: 4)$ 重结晶, 得到白色粉末 $0.21 \mathrm{~g}, \mathrm{TLC}$ 检测为一个点, 产率为 $90 \%$, 为化合物 $\mathbf{6}$. m.p. $228 \sim 231{ }^{\circ} \mathrm{C} ;{ }^{1} \mathrm{H}$ NMR $\left(500 \mathrm{MHz}, \mathrm{CDCl}_{3}\right.$ ) $\delta: 1.09$ (s, $\left.36 \mathrm{H}, \mathrm{C}\left(\mathrm{CH}_{3}\right)_{3}\right), 1.24\left(\mathrm{~s}, 36 \mathrm{H}, \mathrm{C}\left(\mathrm{CH}_{3}\right)_{3}\right), 2.84(\mathrm{~s}, 16 \mathrm{H}$, $\mathrm{CH}_{2} \mathrm{CO}$ ), 3.46 (d, $\left.J=15.0 \mathrm{~Hz}, 8 \mathrm{H}, \mathrm{ArCH}_{2} \mathrm{Ar}\right), 4.28$ (d, $J=$ $15.0 \mathrm{~Hz}, 8 \mathrm{H}, \mathrm{ArCH}_{2} \mathrm{Ar}$ ), 4.79 (s, 8H, $\mathrm{ArOCH}_{2}$ ), 6.90 (s, 8H, ArH), 7.10 (s, 8H, ArH), 7.50 (s, 4H, NH), 10.86 (bs, 4H, NH); IR (KBr) v: $3437(\mathrm{NH}), 1732(\mathrm{C}=\mathrm{O}), 1627(\mathrm{C}=$ O) $\mathrm{cm}^{-1}$; MS m/z (\%): $1912.1\left(\mathrm{M}^{+}, 100\right)$. Anal. calcd for $\mathrm{C}_{112} \mathrm{H}_{136} \mathrm{~N}_{8} \mathrm{O}_{20}$ : C 70.27, H 7.16, N 5.85; found C 70.19, H 7.24, N 5.77 .

\section{$1.2 .4 \quad 1,2-3,4$-双桥联杯[4]芳烃 5 的合成}

氮气保护下，向干燥的 $200 \mathrm{~mL}$ 三口瓶中依次加入 $200 \mathrm{~mL}$ 干燥二氯甲烷，杯[4]芳烃衍生物 4 (0.25 g, 0.25 $\mathrm{mmol}), N, N^{\prime}$-二环已基碳二亚胺(DCC) $(0.36 \mathrm{~g}, 1.74$ $\mathrm{mmol}$ )和 4-二甲氨基吡啶(DMAP) (0.15 g, $1.22 \mathrm{mmol}$ ), 
室温下反应 $5 \mathrm{~h}$, 用 TLC 检测跟踪至原料消失. 反应液 室温减压浓缩至 $15 \mathrm{~mL}$ 左右, 加入 $15 \mathrm{~mL}$ 蒸馏水摚拌, 静置分液, 有机层干燥减压浓缩至 $2 \sim 3 \mathrm{~mL}$. 残余物用 快速色谱柱(硅胶 100 200 目, 淋洗剂为石油醚/1,2-二 氯乙烷, $V: V=1: 1)$ 分离, 得到白色粉末化合物 $\mathbf{5}$ $(0.062 \mathrm{~g})$ 和 $6(0.14 \mathrm{~g})$, 产率分别为 26\%和 58\%. 5: m.p. $287 \sim 289{ }^{\circ} \mathrm{C} ;{ }^{1} \mathrm{H}$ NMR $\left(500 \mathrm{MHz}, \mathrm{CDCl}_{3}\right) \delta: 1.09(\mathrm{~s}, 18 \mathrm{H}$, $\left.\mathrm{C}\left(\mathrm{CH}_{3}\right)_{3}\right), 1.24$ (s, $\left.18 \mathrm{H}, \mathrm{C}\left(\mathrm{CH}_{3}\right)_{3}\right), 2.83\left(\mathrm{~s}, 8 \mathrm{H}, \mathrm{CH}_{2} \mathrm{CO}\right)$, 3.47 (d, $J=15.0 \mathrm{~Hz}, 4 \mathrm{H}, \mathrm{ArCH}_{2} \mathrm{Ar}$ ), 4.29 (d, $J=15.0 \mathrm{~Hz}$, $\left.4 \mathrm{H}, \mathrm{ArCH}_{2} \mathrm{Ar}\right), 4.78$ (s, 4H, $\left.\mathrm{ArOCH}_{2}\right), 6.90$ (s, 4H, ArH), 7.09 (s, 4H, ArH), 7.51 (s, 2H, NH), 10.85 (bs, 2H, NH); IR (KBr): $3447(\mathrm{NH}), 1732(\mathrm{C}=\mathrm{O}), 1628(\mathrm{C}=\mathrm{O}) \mathrm{cm}^{-1}$; MS $m / z$ (\%): $956.7\left(\mathrm{M}^{+}, 100\right)$. Anal. calcd for $\mathrm{C}_{56} \mathrm{H}_{68} \mathrm{~N}_{4} \mathrm{O}_{10}$ : C 70.27, H 7.16, N 5.85; found C 70.21, H $7.23, \mathrm{~N} 5.72$.

\section{References}

[1] Nabeshima, T.; Saiki, T.; Sumitomo, K. Tetrahedron Lett. 2004, 45, 4719 .

[2] Bhalla, V.; Kumar, M.; Katagiri, H.; Hattori, T.; Miyano, S. Tetrahedron Lett. 2005, 46, 121.

[3] Joseph, R.; Rao, C. P. Chem. Rev. 2011, 111, 4658.

[4] Li, X.; Gong, S. L.; Zhang, C. L.; Zheng, Q.; Chen, Y. Y. Tetrahedron Lett. 2006, 47, 7695.
[5] Tantrakarn, K.; Ratanatawanate, C.; Pinsuk, T.; Chailapakul, O.; Tuntulani, T. Tetrahedron Lett. 2003, 44, 33.

[6] Pérez-Casas, C.; Rahman, S.; Xi, Z.; Yamato, T. J. Inclusion Phenom. Macrocyclic Chem. 2007, 58, 383.

[7] Štastný, V.; Stibor, I.; Dvoáková, H.; Lhoták, P. Tetrahedron 2004, $60,3383$.

[8] Yang, F.; Zhao, X.; Guo, H.; Lin, J.; Liu, C. J. Inclusion Phenom. Macrocyclic Chem. 2008, 61, 139.

[9] Yang, F.; Ji, Y.; Zheng, L.; Guo, H.; Lin, J. Supramol. Chem. 2006, $18,177$.

[10] Yang, F.; Zhao, X.; Huang, C.; Guo, H.; Zheng, S.; Peng, Q. Chin. Chem. Lett. 2006, 17, 1029.

[11] Budka, J.; Lhoták, P.; Stibor, I.; Michlová, V.; Sykora, J.; Cisarová, I. Tetrahedron Lett. 2002, 43, 2857.

[12] Matthews, S. E.; Schmitt, P.; Felix, V.; Drew, M.; Beer, P. D. J. Am. Chem. Soc. 2002, 124, 1341.

[13] Yang, F.; Liu, L.; Liu, C.; Zheng, X.; Guo, Y. Chin. Chem. Lett. 2008, 19, 9 .

[14] Chawla, H. M.; Singh, S. P.; Upreti, S. Tetrahedron 2007, 63, 5636.

[15] Lhoták, P.; Štastný, V.; Stibor, I.; Sýkora, J. Tetrahedron 2005, 61, 9990.

[16] Düker, M. H.; Gómez, R.; Velde, C. V.; Azov, V. A. Tetrahedron Lett. 2011, 52, 2881.

[17] Yang, F.; Wang, Y.; Guo, H.; Xie, J.; Liu, Z. Can. J. Chem. 2010, 88,622 .

[18] Yang, F.; Zheng, L.; Ji, Y.; Guo, H.; Lin, J. Chin. J. Org. Chem. 2006, 26, 268 (in Chinese).

(杨发福, 郑林禄, 季衍卿, 郭红玉, 林建荣, 有机化学, 2006, 26, 268.) 